\title{
Structure-Based Coarse- and Fine-Graining in Soft Matter Simulations
}

\author{
Nico F.A. van der Vegt, Christine Peter, and Kurt Kremer \\ Max Planck Institute for Polymer Research
}

\section{CONTENTS}

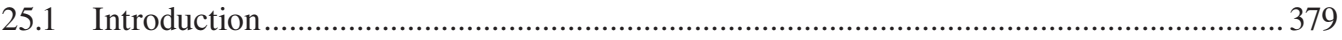

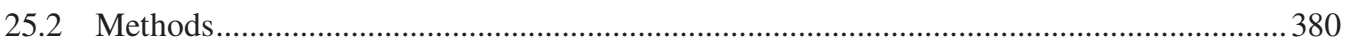

25.2.1 General Concept ......................................................................................... 380

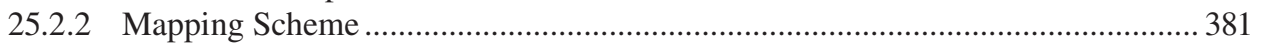

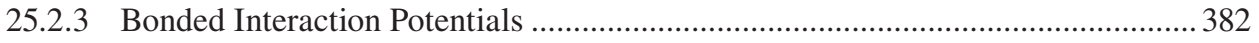

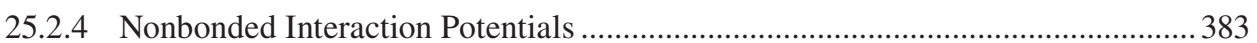

25.2.5 Coarse-Grained Simulations: Equilibration of Mesoscale Structures ......................384

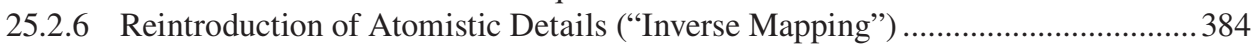

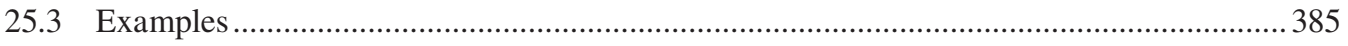

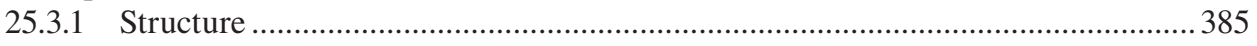

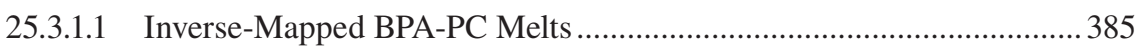

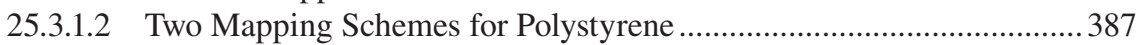

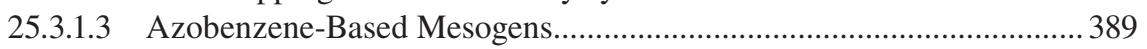

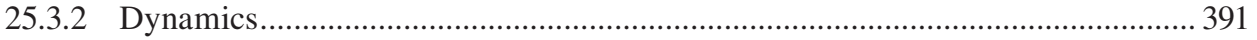

25.3.2.1 Long-Time Atomistic BPA-PC Trajectories Obtained by Inverse Mapping................................................................................... 391

25.3.2.2 Dynamic Speedup: Additive Molecules in a Long-Chain

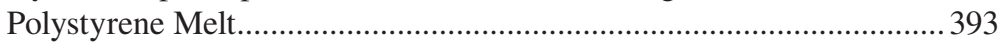

25.4 Some Recent Developments and Future Perspectives ................................................... 394

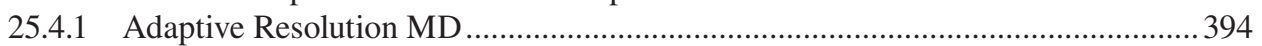

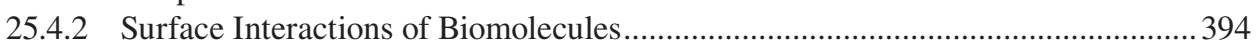

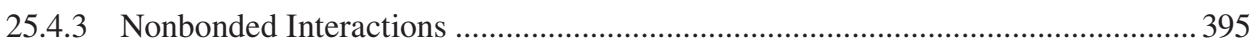

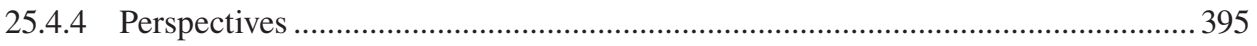

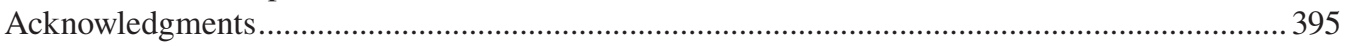

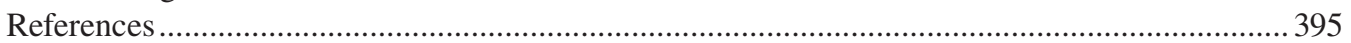

\subsection{INTRODUCTION}

Many physical phenomena in biology, chemistry, and materials science involve processes occurring on atomistic length and time scales, which affect structural and dynamical properties on mesoscopic scales exceeding far beyond atomistic ones. Because it is infeasible (and most often undesirable) to run computer simulations of very large systems with atomically detailed models, mesoscale (coarse-grained) models are being developed through which structural relaxations can 
be studied at large length scales, allowing for full system equilibration on mesoscopic time scales [1-5]. Ideally, coarse-grained (CG) models stay reasonably close to the chemical structure of the material so that inverse-mapping (reintroduction of chemical details) procedures can be employed and atomically detailed processes can be studied in various windows of the CG trajectory where "something interesting happens." Only with that possibility at hand, the corresponding CG models can be used to describe chemically realistic systems over a wide range of length and times scales in a hierarchical, sequential set of simulations at multiple resolution levels, or in a single, multiscale simulation where the level of resolution can be changed at will, locally or adaptively (in the course of a simulation).

Linking chemical structure to properties and behavior of materials on different time and length scales can be achieved only if the various (high- and low-) resolution models involved are structurally consistent. Ideally, the structural agreement should hold down to the smallest possible length scale, which is the dimension of a CG unit. It is important to realize that, depending on the extent of coarse graining, many all-atom (AT) states correspond to one CG configuration. Although a oneto-one correspondence between AT and CG configurations therefore does not exist, it is crucial that the conformational ensemble obtained with a CG model corresponds to that of the all-atom system, with the latter being analyzed in terms of the CG degrees of freedom. If we limit ourselves to the classical (non-quantum mechanical) case it means that the CG model must be parameterized such that the statistical weights of CG configurations are obtained from a (Boltzmann) weighted average over all corresponding AT states. Although for many systems we are still far from achieving this goal, it makes clear that quantum mechanical (QM), classical atomistic (AT) and coarse-grained (CG) mesoscopic models should ideally be developed such that "scale-hopping" [1,6-8] is possible in both forward and backward directions. It is the purpose of this chapter to discuss some of these issues and provide examples of CG models and multiscale modeling methods recently developed in our lab. We will emphasize structure-based coarse graining for reasons following from the goal to allow for structure-based scale hopping as outlined above. In doing so, we follow a coarsegraining prescription without using ad hoc input in order to get the desired properties right. Alternative coarse-graining approaches (described elsewhere in this book) will not be discussed. Also, approaches that go much further and map the whole chain to one ellipsoidal [9] particle or just a soft sphere [10] are not considered here.

Figure 25.1 shows the systems that are discussed in this chapter. It includes bisphenol-A polycarbonate (BPA-PC) [11,12], polystyrene (PS) [13,14], and the liquid crystalline (LC) azobenzene derivative $8 \mathrm{AB} 8$ [15]. The CG representations are superimposed onto the chemical structures illustrating the typical level of coarse graining. In Section 25.2 we shall discuss the coarse graining and inverse-mapping procedures employed. In Section 25.3, several aspects of the CG models representing the above molecules are being discussed in terms of the structure (melt structure, chain conformations, LC order) and dynamics they predict. In this section, we focus on recent developments (what can be done nowadays with structure-based coarse-graining approaches and where possible pitfalls are that need to be avoided), inverse-mapped atomistic structures, and issues concerning the time-mapping procedure. In Section 25.4, an outlook to future developments and recent extensions to an adaptive scheme is being presented.

\subsection{METHODS}

\subsubsection{General Concept}

The following section will be organized along the sequence of steps in deriving a CG model: first, we have to formulate a mapping scheme that relates the coordinates in the atomistic description with the centers of the CG particles. Second, one has to decide on a strategy concerning bonded and nonbonded interactions. In the coarse-graining procedure used by us, nonbonded and bonded interactions are strictly separated and derived sequentially. Such a clear separation makes the possibility 


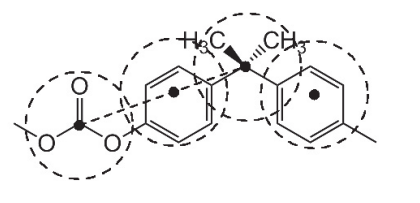

BPA-PC

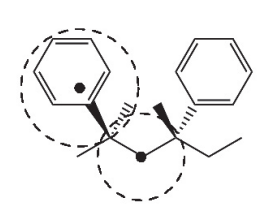

Scheme I

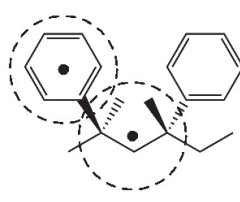

Scheme II

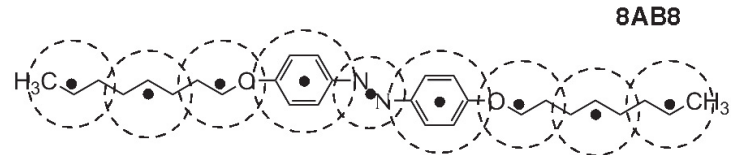

FIGURE 25.1 Atomistic and coarse-grained models of bisphenol-A-polycarbonate (BPA-PC), polystyrene (PS), and 4,4'-dioctyloxyazobenzene (8AB8). The CG mapping points are indicated with black dots. The corresponding CG superatoms, centered on the CG mapping points, are represented by the dashed spheres. For PS, two mapping schemes are shown. For BPA-PC, mapping points on the carbonate, phenyl, and isopropylidene groups are connected through a single CG bond.

to transfer potentials more likely, and allows us to distinguish between effects due to inter- and intramolecular potentials. Consequently, we describe separately how bond stretching, bond angle bending, and dihedral torsion potentials in the CG scheme are derived based on an atomistically detailed simulation of the isolated molecule in vacuo. Next, nonbonded interaction potentials between CG beads are derived based on the liquid structure of polymer melts or low-molecular-weight fluids (i.e., fragments of the target molecule or chain). These interaction potentials are subsequently used to generate well-equilibrated mesoscale structures and long-time trajectories of the system of interest. A last step, which also belongs to the coarse-graining procedure in the sense that it is a crucial link between the atomistic and the CG level of resolution, is the procedure of reintroducing atomistic details into a CG simulation trajectory ("back-mapping" or "inverse mapping").

\subsubsection{Mapping Scheme}

The mapping scheme relates the atomistic coordinates of a structure to the bead positions in the CG model. (Our models usually rely on CG centers with spherically isotropic potentials.) It is clear that there is no unique way to map a given set of atoms onto a coarser description. However, depending on the specific system and on the properties of the system that one wants to see reflected on the coarse level, one can define criteria to determine mapping points. Examples for such criteria are requirements to keep the ability to account for stereoregularity of chain molecules (e.g., PS [13,14]), or to capture certain geometry changes. For example, for azobenzene containing LCs (8AB8) [15] one needs a clear distinction between the cis and trans geometry of the AB unit if one wants to investigate photoinduced phase transitions. There are other criteria that make a certain CG model more or less appealing, for example, in the PS example, a mapping was chosen which avoids "branching off" dangling side groups; that is, all CG beads are linearly connected in the chain, which saves complicated torsion and angle potentials $[13,14]$.

When discussing the computational efficiency of a specific mapping scheme, one has to take several aspects into account. Trivially one would assume that fewer CG beads per molecule result in higher computational efficiency. In addition to a reduction in number of degrees of freedom 
(DOFs), there is a speedup of the dynamics of the system due to a reduced molecular friction (larger beads, smoother potentials) of the CG model. In the case of chain molecules, there is, however, another aspect that should be kept in mind. Chain dynamics is faster if the envelope of the beads of the chain is tube-like, preventing optimised sphere packing and subsequent cage formation with corresponding higher friction [16]. A measure for this commensurability is given by the ratio of mean bond length and bead diameter. This criterion was used to explain why, for BPA-PC, a mapping scheme of figure 25.1 with more beads is computationally more efficient than another one where the phenyl rings were included in somewhat larger spheres at the carbonate and isopropylidene units [11].

Another criterion that needs to be accounted for when devising a mapping scheme relates to the statistical correlations of internal DOFs. The mapping should be chosen such that these correlations are as weak as possible so that the intramolecular (bonded) potentials can be separated into bond stretching, bond angle bending, and torsion terms, as outlined in the next subsection.

\subsubsection{Bonded Interaction Potentials}

First of all, the determination of interaction potentials for the CG model is based on the assumption that the total potential energy $U^{\mathrm{CG}}$ can be separated into bonded/covalent $\left(U_{\mathrm{B}}^{\mathrm{CG}}\right)$ and nonbonded $\left(U_{\mathrm{NB}}^{\mathrm{CG}}\right)$ contributions [1]:

$$
U^{\mathrm{CG}}=\sum U_{\mathrm{B}}^{\mathrm{CG}}+\sum U_{\mathrm{NB}}^{\mathrm{CG}}
$$

Intramolecular bonded/covalent interactions of the CG model are determined by sampling the distributions of (CG) conformational DOFs based on an atomically detailed simulation (Monte Carlo or molecular dynamics (MD) using a stochastic thermostat to ensure proper equilibration) of an isolated molecule in vacuo. These conformational distributions are in general characterized by CG bond lengths $\{r\}$, bond angles $\{\theta\}$, and torsions $\{\phi\}$; that is, $P^{\mathrm{CG}}(r, \theta, \phi, T)$ and are clearly temperature dependent (for simplicity we assume here that there is only one kind of bond, bond angle, or torsion). If one assumes that the different $\mathrm{CG}$ internal DOFs are uncorrelated, $P^{\mathrm{CG}}(r, \theta, \phi, T)$ factorizes into independent probability distributions of bond length, angle, and torsional DOFs:

$$
P^{\mathrm{CG}}(r, \theta, \phi, T)=P^{\mathrm{CG}}(r, T) P^{\mathrm{CG}}(\theta, T) P^{\mathrm{CG}}(\phi, T) .
$$

This assumption has to be carefully checked (it is not uncommon that CG DOFs are correlated, for example that certain combinations of CG bonds, angles, and torsions are "forbidden" in the distributions obtained from the "real" atomistic chain), and is an important test of the suitability of a mapping scheme [14], because a mapping scheme that requires complex multiparameter potentials is computationally rather inefficient.

The individual probability distributions $P^{\mathrm{CG}}(r, T), P^{\mathrm{CG}}(\theta, T)$, and $P^{\mathrm{CG}}(\phi, T)$ are then Boltzmann inverted to obtain the corresponding potentials and-through taking the derivativesthe forces

$$
\begin{aligned}
& U^{\mathrm{CG}}(r, T)=-k_{\mathrm{B}} T \ln \left[P^{\mathrm{CG}}(r, T) / r^{2}\right]+C_{r}, \\
& U^{\mathrm{CG}}(\theta, T)=-k_{\mathrm{B}} T \ln \left[P^{\mathrm{CG}}(\theta, T) / \sin \theta\right]+C_{\theta}, \\
& U^{\mathrm{CG}}(\phi, T)=-k_{\mathrm{B}} T \ln P^{\mathrm{CG}}(\phi, T)+C_{\phi} .
\end{aligned}
$$


When deriving potentials from bond and angle distributions one has to account for the respective volume elements $r^{2}$ and $\sin \theta$. Using the inverted distributions as potentials means that these potentials are in fact potentials of mean force. Ergo they are free energies and consequently temperature dependent. As mentioned before, this temperature dependence originates not only from the prefactor $k_{\mathrm{B}} T$, but from the distributions $P$ themselves. Strictly speaking they can only be applied at the temperature (state point) they were derived at.

The approach outlined in this section is in contrast to other approaches, where the CG internal DOFs are determined based on the distributions obtained from an atomistic simulation of the liquid phase [3]. In the latter case one obtains potentials for bonded and nonbonded interactions simultaneously from the same liquid simulation; consequently they are potentially interdependent; that is, there is no clear separation between covalent and nonbonded interaction potentials. We achieve this separation by deriving CG bond length, bond angle, and torsional distributions from the atomically detailed conformations sampled by a single (chain) molecule in vacuo. In the atomistic simulation performed to generate the distributions of CG intramolecular DOFs, the inclusion of nonbonded interactions has to be taken with care to avoid "double counting" of interactions. This means that long-range intrachain nonbonded interactions (beyond the distance between CG beads which are explicitly covered via bonded interaction potentials, for example, beyond the distance of three CG bonds if torsion potentials are used) should be excluded when the single chains are sampled. Instead these long-range interactions should be treated equivalently to CG intermolecular nonbonded interactions.

\subsubsection{Nonbonded Interaction Potentials}

The general principle when deriving nonbonded interaction potentials is to reproduce structural properties; that is, radial distribution functions of (low-molecular-weight) liquids or polymer melts (experimentally known or obtained from atomistic simulations). Similarly to the above case of bonded interaction functions, one has two principal options: either (1) to use analytical potentials, in which case one would optimize the parameters of a chosen analytical function to reproduce the structure of the atomistic melt/liquid as accurately as possible (or to account for the excluded volume interaction only, in which case no further optimization is being done, see BPA-PC [1,11]); or (2) one would use numerically derived tabulated potentials, which are designed such that the CG liquid reproduces the atomistic liquid structure, when the latter is analyzed in terms of the overlaid CG structure the microstate corresponds to.

In the first case, analytical potentials of various types can be used: the "normal" Lennard-Jones 12-6 potential is frequently used; it has, however, been proven to be in many cases too steeply repulsive; that is, too "hard," for CG particles, which are rather large and soft. In that case, softer LennardJones-type (e.g., 9-6 or 7-6) [14], Buckingham or Morse potentials [15] are employed. These potentials are usually made purely repulsive in the spirit of the WCA potential [17] by shifting upwards and truncating in the minimum. In order to search in parameter space to optimize these analytical potentials to reproduce a given liquid or melt structure, a simplex algorithm can be used $[18,19]$.

Concerning the second option to generate numerically a tabulated potential that closely reproduces a given melt structure; that is, a given radial distribution function $g(r)$, the iterative Boltzmann inversion method has been developed [20,21]. This method relies on an initial guess for a nonbonded potential $U_{\mathrm{NB}, 0}^{\mathrm{CG}}$. Usually the Boltzmann inverse of the target $g_{\text {target }}(r)$; that is, the potential of mean force,

$$
U_{\mathrm{NB}, 0}^{\mathrm{CG}}=-k_{\mathrm{B}} T \ln g_{\text {target }}(r),
$$

is used, with which one then generates a CG simulation trajectory of the liquid. The resulting structure will not match the target structure since, due to multibody interactions, the potential of mean 
force is a good estimate for the pair potential only at very high dilution. However, using the iteration scheme

$$
U_{\mathrm{NB}, i+1}^{\mathrm{CG}}=U_{\mathrm{NB}, i}^{\mathrm{CG}}+k_{\mathrm{B}} T \ln \left[\frac{g_{i}(r)}{g_{\text {target }}(r)}\right],
$$

the original guess can be self-consistently refined until the desired structure is obtained. There can be limits to this approach because it is not always clear whether the chosen CG mapping scheme can converge to an optimal fit.

For complex molecules with a large number of different CG beads or more importantly in the case of molecules that form complex or anisotropic liquid or melt structures, for example, liquid crystals, the procedure to determine nonbonded interaction functions is more complicated. In these cases it is advantageous to split the target molecule into fragments so that the nonbonded interactions between different bead types can be determined based on the structure of isotropic liquids of these fragment molecules. One principal problem that arises if one uses smaller fragments to generate nonbonded interaction potentials for larger molecules is that different conformations may contribute to the structure of the liquid of the fragment molecules differently than in the (polymeric) melt [22]. One example where such an effect may play a role is in the parameterization of phenyl rings based on the structure of liquid benzene: in that case the relative population of parallel and perpendicular arrangements of two phenyl rings that are part of longer chain molecules potentially differs from the arrangements in liquid benzene for steric reasons. Despite these potential problems, the procedure to parameterize CG nonbonded interactions based on small molecules is promising to generate $\mathrm{CG}$ parameters for complex molecules and it also allows reuse of certain CG potentials for reoccurring building blocks (such as alkyl or phenyl groups), which aims at some sort of building block or LEGO set of molecule fragments for CG simulations. Of course, this approach needs to be carefully tested and the transferability of the potentials generated from these fragments to (slightly) different conditions needs to be carefully evaluated (as will be further discussed in the Examples section).

\subsubsection{Coarse-Grained Simulations: Equilibration of Mesoscale Structures}

Even with the dynamic speedup gained by CG models, it is not trivial to obtain well-equilibrated structures of mesoscale (polymeric) systems. In particular for long-chain molecules (beyond a few entanglement lengths), branched polymers, or polymers at interfaces, brute force MD algorithms that follow the slow dynamics of the system will not easily lead to complete equilibration of the chains. Besides, criteria are needed to judge whether a melt structure is really equilibrated since local monomer packing and the statistics of end-to-end distances or radii of gyration are not sufficient. Auhl et al. [23] describe such criteria and investigate various methods to generate wellequilibrated polymer melts using MD simulations. Based on such CG structures and simulation trajectories it is in the next step possible to reintroduce atomistic coordinates and to obtain equilibrated atomistic structures on the mesoscale or long-time atomistic trajectories.

\subsubsection{Reintroduction of Atomistic Detalls ("Inverse Mapping")}

Inverse mapping; that is, reintroduction of atomistic detail, requires finding a set of atomistic coordinates that corresponds to a given CG structure. In general there is no unique solution to that problem since each CG structure corresponds to many all-atom configurations. Therefore, one needs to find one representative all-atom structure, with the correct statistical weight of those DOFs that are not resolved in the CG description. Several slightly different strategies to reintroduce atomistic detail into a CG structure have been presented [2,3,12,13,15,24]. 
If the (polymer) chain consists of reasonably rigid (all-atom) fragments, it is sufficient to fit these rigid all-atom units onto the corresponding CG chain segment coordinates. The atomistic fragments can be taken from a pool of structures that correctly reflect the statistical weight of those DOFs (certain torsions, ring flips, etc.) that are not resolved in the CG description and that relax too slowly to be properly equilibrated in a short equilibration run of the resulting atomistic structure.

If the CG molecule/polymer chain consists of very flexible units, for example, alkyl tails, and in particular if the CG structure consists of small molecules (8AB8, a low-molecular-weight LC), where even in a very short equilibration step, the atomistic structure significantly diffuses away from the CG coordinates, a slightly different strategy was employed: atomistic coordinates were inserted into the CG structure using fragments for the rigid units and random atomistic positions for the flexible units (with the constraint that the atomistic coordinates have to satisfy the "mapping" condition; that is, the atomistic coordinates have to correspond to the CG structure if one applies the mapping scheme). The resulting structure was then relaxed (energy minimized and equilibrated by MD simulations), while restraining the atomistic coordinates to CG mapping points. This results in a perfectly equilibrated structure that (almost, depending on the strength of the restraining potential) exactly reproduces the CG structure.

\subsection{EXAMPLES}

In this section, we discuss, on the basis of the three examples shown in figure 25.1 (and 25.6), various aspects of structure-based coarse graining focusing on recent developments, inverse-mapped atomistic structures and dynamics. In Section 25.3.1 ("Structure") we discuss experimental validation of inverse-mapped BPA-PC and PS melt structures and the prospects that open up due to the resulting well equilibrated long-time/large-scale atomistic trajectories; we illustrate the consequences of the choice of a CG mapping scheme using the example of PS, and we show the application of the present coarse-graining approach to LC molecules. In Section 25.3.2 ("Dynamics") we discuss how, by application of CG models, the corresponding time scales are modified. In that context we compare BPA-PC chain dynamics in all-atom and CG molecular liquids as well as diffusion of low-molecularweight additives in CG PS melts.

\subsubsection{Structure}

\subsubsection{Inverse-Mapped BPA-PC Melts}

Although many aspects of, for example, polymer dynamics, overall chain conformations, or LC order can be well described with CG resolution, for many other questions chemical details need to be reintroduced by inverse-mapping methods described in the previous section. This we illustrate here by discussing aspects of packing of BPA-PC polymeric liquids $[2,12]$ and the evaluation of interactions (chemical potentials) of small molecules inside polymeric microstructures [25].

To check the quality of BPA-PC melt structures, we calculated neutron scattering functions of the (reintroduced) all-atom melts. Figure 25.2a shows the coherent neutron scattering function for a melt containing 100 chains of $N=20$ chemical repeat units at two temperatures [12,26]. The simulated functions are compared with experiments obtained at $T=1.5 \mathrm{~K}$ [26] and consequently most probably a slightly higher density. The peak at $0.6 \AA^{-1}$ corresponds to the intrachain sequential carbonatecarbonate distance of about $11 \AA$ and not to interchain correlations. This could be concluded from the simulations, where the n-scattering functions were calculated. For the "computer samples" one can vary the atomic scattering lengths in the analysis and delete or create scattering contrast for any correlation at will. The main peak (amorphous halo) corresponds to the typical interchain (packing) distance. The agreement between the experimental data and the simulations is close to perfect. The discrepancies are due to the higher temperature of the simulated melts, which causes the amorphous halo to broaden and to shift to slightly larger distances and the peak corresponding to intrachain 

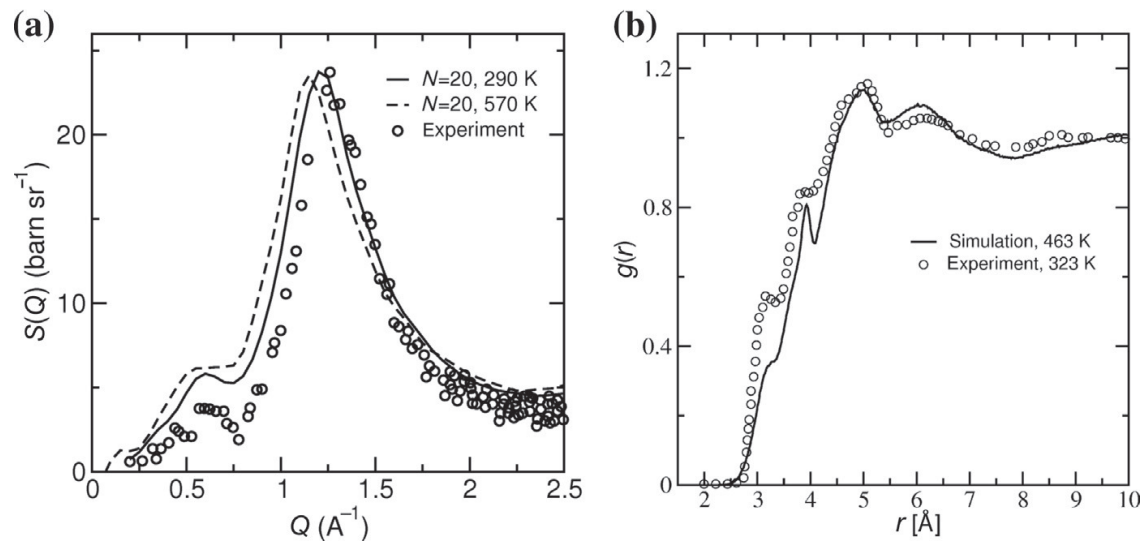

FIGURE 25.2 (a) Coherent neutron scattering function of two BPA-PC melts (290 and $570 \mathrm{~K}$ ) [12] in comparison with experiments of a sample, which was cooled down and kept at a temperature of $1.5 \mathrm{~K}$ [26]. The solid and dashed curves were obtained by inverse mapping of chemical details for a system containing 100 chains of 20 repeat units each. (b) Radial distribution function of a simulated atactic polystyrene melt obtained by inverse mapping of chemical details [13] in comparison with the experimental RDF obtained from X-ray diffraction [27]. All atom-atom correlations are included except those between atoms within phenyl rings and atoms along the backbone separated by less than three chemical bonds.

carbonate-carbonate correlations to wash out. A comparison of simulated scattering curves with experimental data for partially deuterated BPA-PC samples was also made [2,12], which further supported the overall agreement with experiments [26].

A similar comparison was made for a PS melt. Figure 25.2b shows the total radial distribution function obtained after reintroducing chemical details together with experimental data obtained by wide-angle X-ray diffraction measurements [27]. In both the simulation and the experimental data, intramolecular correlations due to 1-2 and 1-3 bonded neighbors (along the backbone) as well as all intraring correlations have been removed in order to emphasize the features deriving from the packing of nonbonded segments. Despite differences in temperature and chain length of the simulated and experimental samples, the overall agreement is very good. Moreover, in our analysis of the simulation trajectories we employed a united-atom model. Because of that, we assumed $Q$ independent atomic scattering functions taking the carbon nuclear positions as scattering centers. This assumption gives rise to a stronger developed peak in the simulated data slightly below $4 \AA$ in comparison with the $\mathrm{X}$-ray experiment.

As a second example we mention a significant advantage of using inverse-mapped polymer microstructures in studying permeation of small molecules (so-called 'penetrants'). The first application using this approach was a computational study of phenol in BPA-PC [28]. The phenol diffusion process revealed a strong coupling between size and shape fluctuations of the pore space and the hopping of the penetrant. The pore structure was also analyzed in terms of the positronium annihilation time [29]. The resulting lifetime distribution functions compared very well to those from experiments, again supporting the overall consistency of the approach. In addition to diffusion, the penetrant solubility or excess chemical potential inside the polymer microstructure is also of interest. With currently available methods, penetrant excess chemical potentials can only be computed with sufficient statistical accuracy for fairly small penetrants. These are usually pure substances, such as gases under ambient conditions. A polymeric simulation box with a typical linear dimension of $4-5 \mathrm{~nm}$ is usually large enough to contain a statistically meaningful number of pre-existing, empty cavities, which can host a small molecule without significantly modifying the matrix. Thus standard methods, such as test-particle insertion techniques, can be used to obtain reliable data. However, calculations of excess chemical potentials of larger penetrants, with equally 
high statistical reliability, are extremely cumbersome for several reasons. Most importantly, larger penetrants (e.g., phenol, propane, chloroform) occupy larger cavities, which in microstructures with the above-mentioned linear dimensions occur very infrequently, albeit contributing significantly to the excess chemical potential. This problem can be resolved only if a large number of statistically uncorrelated microstructures can be generated at small computational expense. Obviously, reinserted all-atom microstructures generated from CG mesoscale simulations can be used to resolve this problem. Based on large systems generated in this way, we currently explore an alternative, nonequilibrium free-energy sampling technique, in order to resolve insertion problems usually encountered with large molecules in dense systems [25].

\subsubsection{Two Mapping Schemes for Polystyrene}

As discussed in Section 25.2, CG intramolecular potentials are developed assuming that the CG bond length, bond angle, and dihedral angle have no interdependencies. The validity of this assumption depends however on how we choose the CG mapping points. Figure 25.1 shows two CG representations for PS [14]. In the first scheme (I), the PS repeat unit is represented by a CG bead (type " $A$ ") localized on the methylene position, and another CG bead (type " $B$ ") is localized on the mass center position of the remaining atoms. $A$ and $B$ beads are connected by CG bonds giving rise to bond angles $\theta_{A B A}$ and $\theta_{B A B}$, and dihedral torsions $\varphi_{A B A B}$ and $\varphi_{B A B A}$. In the second scheme (II), bead $A$ is positioned at the center of mass defined by the methylene group and the two adjacent $\mathrm{CH}$-groups (taking however the half-masses rather than the full $\mathrm{CH}$ masses in defining the $\mathrm{CG}$ bead mass center). Bead $B$ corresponds to the phenyl group. The $A$ and $B$ beads in scheme (II) are also connected by CG bonds, giving rise to the same number of DOF (see fig. 25.3a). We note that the corresponding intramolecular potentials depend on the chain stereoregularity (i.e., the type of dyad [13]), hence the model can in principle be used in simulations of atactic, isotactic, and syndiotactic PS.

The PS conformation on the left-hand side in figure 25.3a is based on CG mapping scheme (I) and is shown to illustrate how the $(\theta, \varphi) C G$ angles are correlated. If the $A$ bead on the left end of the picture is being rotated along the indicated CG bond, the adjacent $B$ bead will also be rotated because these two beads are directly connected through two underlying chemical bonds. This causes variations of $\varphi_{A B A B}$ and $\theta_{B A B}$ to be correlated. Whether at all and to which degree such correlations lead to erroneous conformational sampling in the CG simulations depends on the mapping scheme and needs to be tested to assess the quality of a mapping scheme.

Figure $25.3 \mathrm{~b}$ shows energy diagrams (defined as $-\ln \left[P^{\mathrm{CG}}(\theta, \varphi) / \sin \theta\right]$ ) in a contour map representation for the racemic PS dyad [14]. The bond bending angle $\theta$ corresponds to BAB and the dihedral angle $\varphi$ to ABAB. The diagrams presented in the left part of this figure are obtained from simulations of a single united atom chain and diagrams on the right were obtained with the corresponding CG models. The upper panel corresponds to mapping scheme (I) and the lower panel to mapping scheme (II) (see fig. 25.3a). From the contour maps obtained with the CG models, the $(\theta, \varphi)$ correlation discussed above is lost to some extent. For example, CG scheme (I) has an energy minimum at $\theta \approx 150^{\circ}$ (upper panel, left), which is about $3 k_{\mathrm{B}} T$ deeper than the minimum at $\theta \approx 100^{\circ}$. Therefore, CG model (I) predominantly samples $\theta \approx 150^{\circ}$, independent from the torsion angle $\varphi$, which causes the energy basin at $(\theta, \varphi) \approx\left(100^{\circ}, 240^{\circ}\right)$ observed with the united-atom model (upper panel, left) to shift to a region $(\theta, \varphi) \approx\left(150^{\circ}, 240^{\circ}\right)$ (upper panel, right) hardly ever sampled by the united-atom model.

With mapping scheme (I), the CG model also samples parts in $(\theta, \varphi)$-space not at all accessible by the united-atom model (e.g., $\left(80^{\circ}, 300^{\circ}\right)$ or $\left.\left(80^{\circ}, 30^{\circ}\right)\right)$. These 'forbidden' regions include conformations with excluded volume violations of CG 1-4 interaction sites (methylene units partly overlapping with phenyl groups). These overlaps can be avoided by introducing a special 1-4 nonbonded interaction in the CG model [13]. Noteworthy, CG model (II) clearly performs much better in this respect. Because special 1-4 nonbonded terms are not needed [14], it is also more consistent with 


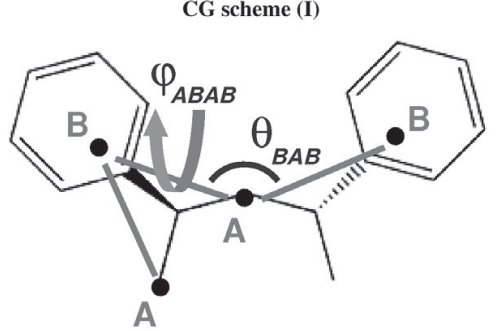

(b)
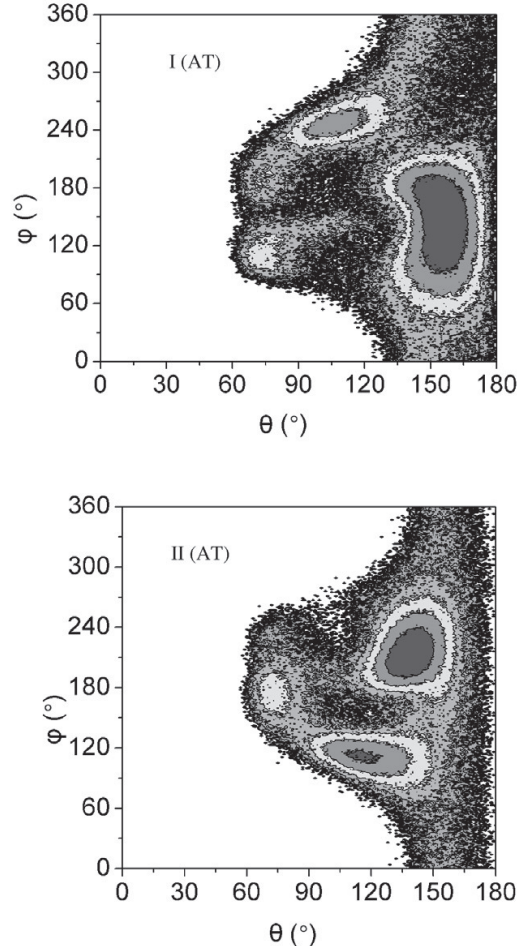

CG scheme (II)
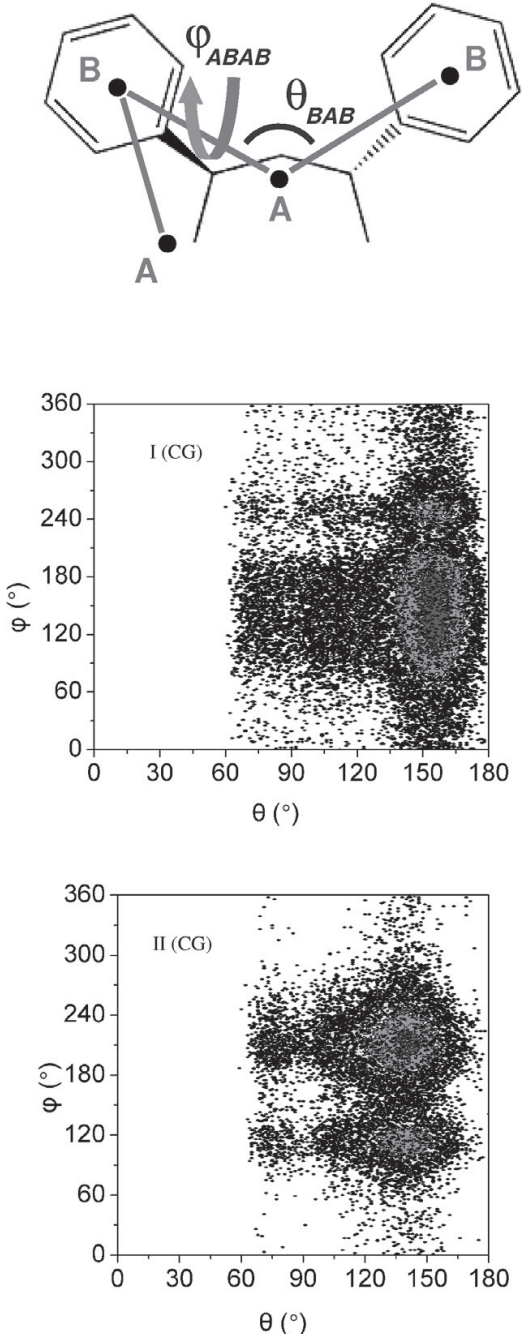

FIGURE 25.3 (a) PS conformation with CG mapping points based on schemes (I) and (II) (cf. fig. 25.1). The CG mapping points are indicated with black dots, CG bonds are indicated by thick gray lines. (b) $(\theta, \varphi)$-energy surfaces: I (AT), obtained by sampling the atomistic model, analyzed in terms of CG scheme (I); I (CG), obtained by sampling with the CG model, scheme (I); II (AT), obtained by sampling the atomistic model, analyzed in terms of CG scheme (II); II (CG), obtained by sampling with the CG model, scheme (II).

the general CG strategy outlined in the previous sections. In addition, there are certain advantages when studying dynamical properties compared to CG model (I).

It is very important to be aware of correlations of internal DOFs in CG simulations, even though artifacts introduced by decoupling the bond-angle bending and dihedral torsion potentials in CG models have so far been shown to affect neither the overall chain conformations nor the ability to successfully perform the inverse mapping in polymer modeling $[13,14]$. This is potentially more problematic in CG models for biomolecules. Here a similar decoupling of the bonded potentials is likely to be more tedious because specific $(\theta, \varphi)$-combinations may turn out to be needed for discriminating turns, helices, sheets, etc. which will be a significant criterion to distinguish "good" and "bad" mapping schemes [30]. 


\subsubsection{Azobenzene-Based Mesogens}

In the previous two examples the coarse-graining procedure (Section 25.2) was applied to polymeric systems, where the behavior of the melt is very much determined through chain connectivity and excluded volume interactions of the polymeric beads. Consequently, it often is not essential to introduce attractive (nonbonded/intermolecular) interactions in order to correctly predict melt structure and dynamics on the mesoscale. It is however very interesting to explore how far the above coarse-graining scheme carries if one tries to apply it to systems where attractive nonbonded interactions are likely to be more important than in amorphous polymers; that is, where the balance of attractions between different chemical units plays a possible role in structure formation. Biopolymers, liquid crystals, and in general self-assembling systems are examples where this can be of importance. The compound 8AB8 (see fig. 25.1) is a LC compound that contains azobenzene as a mesogen and forms a thermotropic nematic phase (and a monotropic smectic). This system is used to study how the coarse-graining approach can be adapted to LC systems. It is of particular interest to build a CG model that is close to an atomistic description not only in order to obtain as much chemical accuracy as possible but also because a close link between the coarse (mesoscale) and the atomistic level is important for multiscale simulation purposes. The reason for this is that azobenzene is a photoswitchable mesogen; that is, it undergoes a trans/cis photoisomerization, which goes along with a drastic shape change: in its trans form it is rod-shaped and functions as a mesogen; in its cis form, it is bent and does not induce a mesophase. Therefore, with 8AB8 a photoinduced nematic-to-isotropic phase transition is observed. This LC phase change and the photoisomerization mechanism are interdependent since on the one hand the LC phase change obviously depends on the degree of trans/cis isomerization, and on the other hand it is believed that the photoisomerization mechanism depends on the (anisotropic) environment or the mechanical pulling of the tails that are attached to the azobenzene group. Therefore, the LC-photoswitching of azobenzene compounds is a true multiscale problem, since the photoisomerization mechanism can be studied using quantummechanical (QM) simulation techniques, whereas investigations of the LC phase change requires much longer length and time scales that can only be achieved by mesoscale (CG) techniques. In this constellation it is important to be able to switch between the levels of resolution, where the atomistic description can function as a link; that is, the coarse model needs to be built on the atomistic description, and the inverse mapping from the CG to the all-atom level is essential to link to QM calculations of the transition.

Ref. 15 describes how a CG model for 8AB8 was developed using the CG techniques developed for polymers. It was shown how intramolecular (bonded) potentials were obtained from simulations of an all-atom single 8AB8 molecule, and how intermolecular potentials were developed based on all-atom simulations of isotropic liquids of fragments of the 8AB8 molecule. The isotropic liquids that were used in the parameterization process were liquid benzene, liquid azobenzene (in its trans and in its cis form), liquid octadecane, and various mixtures of these compounds. Based on the structure of these liquids (radial distribution functions), nonbonded interaction potentials were determined, both using analytical potential functions and the iterative Boltzmann inversion method as detailed in the Methods section (for the case of octadecane see fig. 25.4a). The resulting interaction functions were then used for liquid (trans) 8AB8, where we tried to reproduce the experimentally observed LC phase behavior. In particular we aimed at obtaining a stable nematic phase. One could observe that the use of (soft) analytical potentials that are purely repulsive (in the spirit of the previous coarse-graining examples of polymeric systems) did not yield the correct mesophase behavior of 8AB8; in fact no long-range ordering was observed for the model chosen (see fig. 25.4b), even with a rather wide scan of temperatures and pressures. With potentials generated with the iterative Boltzmann inversion method; that is, numerical (tabulated) potentials which are also partly attractive, it is however possible to generate nematic-like (and smectic) phase of $8 \mathrm{AB} 8$. Thus, for the given molecule; that is, the given size and shape of the mesogen and the given molecular flexibility of the alkoxy tails, it seems to be important to account for attractions 

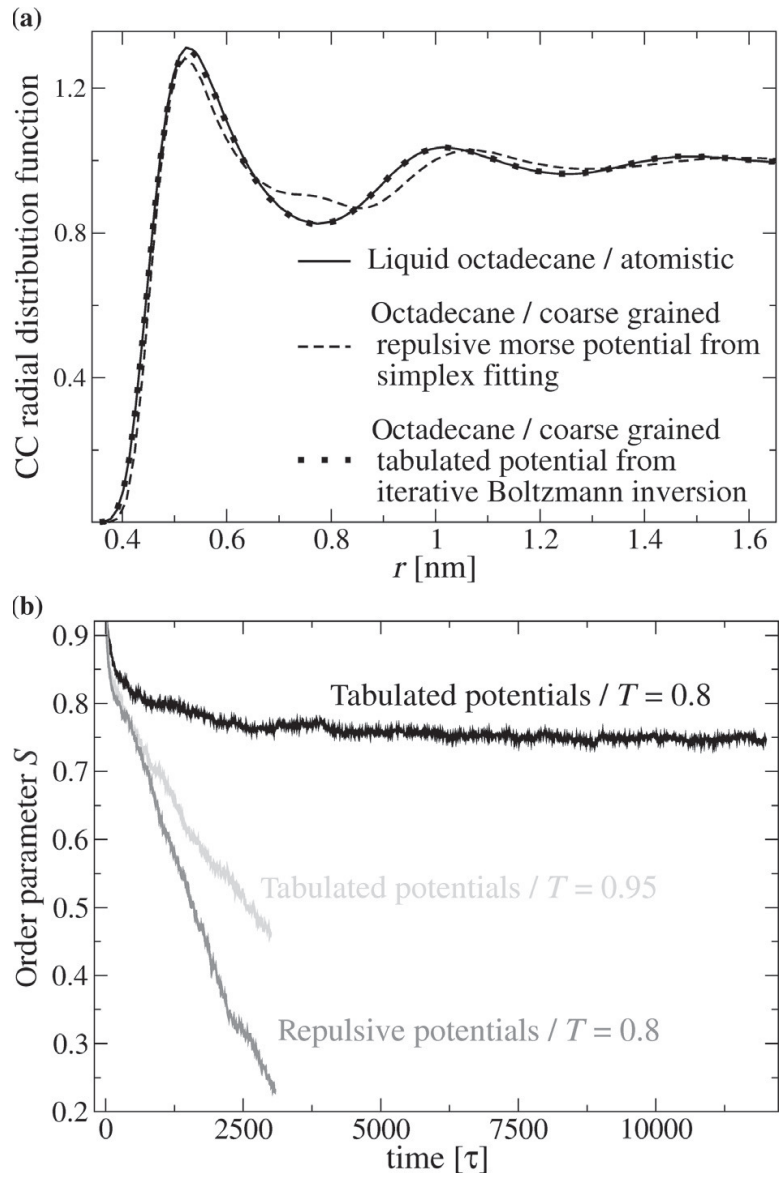

FIGURE 25.4 (a) Structure-based derivation of nonbonded interaction potentials: carbon-carbon radial distribution functions (RDF) of CG centers in an octadecane liquid at $400 \mathrm{~K}$. Thin straight line: RDF obtained from atomistic simulation, mapped onto CG centers. Thin dashed line: RDF obtained in CG simulation after optimizing a purely repulsive Morse potential to reproduce the atomistic structure as well as possible. Fat dotted line: RDF obtained in CG simulation after determining a numerical potential through iterative Boltzmann inversion so that the atomistic structure is reproduced. (b) Order parameter of 8AB8 system in coarse-grained simulations (initial setup fully ordered: four smectic layers). Black and light gray lines: simulations with potentials obtained through iterative Boltzmann inversion (partly attractive). Black line: the system remains ordered at $T=0.8$ (corresponds to $320 \mathrm{~K}$ ) (nematic-like structures are observed). Light gray line: the system becomes isotropic at $T=0.95$ (corresponds to $380 \mathrm{~K}$ ). Dark gray line: Simulation with purely repulsive Morse potentials - the system becomes disordered (at a wide range of temperatures and densities).

between the different beads in the CG model in order to reproduce the ordered phase of $8 \mathrm{AB} 8$. A snapshot of a structure that shows the alignment of the 8AB8 molecules in a nematic-like phase can be seen in the Color Figure 25.6 in the center of the book. This structure was generated by MD simulations using the CG model, the atomistic coordinates that are also shown in the figure were obtained using the inverse-mapping procedure as outlined above (restraining the atom coordinates during equilibration such that the "mapping criterion" is satisfied and the CG structure is therefore preserved). It shows that the structure-based coarse-graining approach originally developed in the polymer framework can be extended to LC systems, where mesoscale (with both large length and long time scales) simulations are essential to probe phase behavior and to generate well equilibrated mesostructures. With the given approach the mesoscale simulations also maintain an 
important link to the chemical structure, and through the inverse-mapping procedure it is possible to obtain atomistic coordinates of the system.

In the course of the parameterization process of the nonbonded interactions, we also performed preliminary tests on the transferability of these fragment-based potentials. We tested, for example, the applicability of potentials derived for pure liquids on mixtures of various compositions and of potentials derived for liquid benzene on liquid trans or cis azobenzene. Overall, the transfer of the nonbonded potentials worked surprisingly well; the limitations are more thoroughly discussed in Ref. 15, and these investigations will also be extended in the future.

\subsubsection{Dynamics}

Within CG models length scales are usually well defined through the construction of the coarse graining itself. In most dynamic CG simulations reported in the literature little attention is paid however to the corresponding "coarse graining" of the time unit. From polymer simulations of both simple continuum as well as lattice models it is known that such simulations reproduce the essential generic features of polymer dynamics; that is, the crossover from the Rouse to the entangled reptation regime, qualitatively and to a certain extent quantitatively [31,32]. While such previous studies concern motion distances on scales well above a typical monomer extension and provide quantitative information on characteristic time ratios, this still leaves a number of open questions. These refer to the predictive quantitative modeling of diffusion, viscosity, rates, and correlation times, etc. of dynamic events as well as to the question of minimal time and length scales CG simulations apply to. Particle mass, size, and energy scale, which are all well defined within a CG model, of course trivially fix a time scale, too, and it is indeed this time scale that is most often reported in MD simulations of CG systems. However, it does not usually correspond to the true physical time scale, because part of the friction experienced by a (sub)molecule (in the AT representation) is lost in the CG representation, causing the CG system to evolve faster. (Note that this is in principle also the case for atomistic simulations that make use of so-called united atoms where aliphatic hydrogen atoms are incorporated into the carbon atoms.) In other words, the fluctuating random forces of atomic DOFs, which are integrated out in the CG model, contribute to a "background friction" that must be considered in order to obtain a realistic time scale in the CG dynamics simulation. In their study of CG blob dynamics in polyethylene melts, Padding and Briels [33] employed effective potentials, frictions, and random forces all derived from detailed MD simulations. Izvekov and Voth [34] proposed a closely related recipe within the coarse-graining framework of force matching. Alternatively, CG dynamic quantities can in some cases be mapped directly onto the corresponding quantity obtained from detailed MD simulations or from experiments. For example, a diffusion coefficient $D^{\mathrm{CG}}$ in units $\left[\mathrm{m}^{2} / \tau\right]$ can be mapped onto the diffusion coefficient $D^{\mathrm{AT}}$ in units $\left[\mathrm{m}^{2} / \mathrm{s}\right]$ providing the time units of the CG simulation $\tau=x[\mathrm{sec}]$. Alternatively, the CG mean squared displacement curve can be superimposed with the atomistic curve at (for atomistic simulations) long times [35]. This approach was used to study entangled polycarbonate (BPA-PC) melts of up to 20 entanglement lengths. The CG simulations provided truly quantitative information on the different measures of the entanglement molecular weight (from displacements, scattering functions, modulus and topological analysis) and the ratios of the different crossover times.

\subsubsection{Long-Time Atomistic BPA-PC Trajectories Obtained by Inverse Mapping}

All CG mapping schemes shown in figure 25.1 stay close to the atomistic structure of the molecules. Therefore, the dynamics of the CG system is expected to follow quite closely that of the atomistic system down to small length and time scales. Moreover, due to significant dynamic speedup, the CG systems can be simulated up to times that exceed far beyond what is possible in brute force detailed atomistic simulations, allowing for in silico experiments looking at exactly the same quantities as in experiments. The idea is to reintroduce atomic details in long-time CG trajectories of the system (BPA-PC for the present case) and measure dynamic relaxations on time scales that altogether cover 
at least nine decades and overlap the experimental regime probed, for example, with spectroscopic techniques. Here we only discuss the dynamic chain scattering function $S(Q, t)$ as obtained in neutron spin echo experiments:

$$
S(Q, t)=\frac{1}{n}\left\langle\sum_{i, j} l_{i} l_{j} \exp \left[i \mathbf{Q} \cdot\left(\mathbf{r}_{i}(t)-\mathbf{r}_{j}(0)\right)\right]\right\rangle_{|\mathbf{Q}|} .
$$

The double sum runs otver all $n$ atoms in the chain. The term $\mathbf{r}_{i}$ is the position of atom $i$ and $l_{i}$ is the neutron scattering length of atom $i$. The index $|\mathbf{Q}|$ indicates spherical averaging. For nonentangled melts on time scales above the local fast oscillations and above the persistence length of the polymer the Rouse model predicts $S(Q, t) / S(Q, 0) \propto \exp \left(-W Q^{2} t^{1 / 2}\right)$, where $W$ is related to the effective bead friction. The onset of this universal behavior is typically small compared to the diffusion time and chain extension. For larger times the overall diffusion takes over; that is, $S(Q, t) / S(Q, 0) \propto \exp \left(-D Q^{2} t\right)$. In the case of entangled polymers, $S(Q, t)$ displays a qualitatively different behavior due to the tube-like confinement of the monomer motion. On intermediate time scales the scatterer "sees" a smeared-out monomer density in the tube of diameter $d_{T}$ leading to an analog of a Debye Waller factor with, in the simplest approximation $S(Q, t) / S(Q, 0)=1-Q^{2} d_{T}^{2} / 36$. CG and atomistic MD simulations of BPA-PC melts were performed with $N=5$ up to $N=120$ repeat units [35] and used to analyze this property. The entanglement molecular weight of BPA-PC (1200-1400 g/mol) corresponds to $N_{e} \approx 5-6$ repeat units. Based on performing a time mapping by superimposing repeat unit mean squared displacements of the $\mathrm{CG}$ and atomistic systems for $N=5$ and $N=20$ for long times, a time unit is obtained. While the intrinsic time unit of the CG model (determined through conversion of Lennard-Jones reduced units, assuming the same mass for all beads) is $\tau \approx 1.7 \mathrm{ps}$, the physical time unit of the underlying BPA-PC is much larger, namely $\tau=30 \mathrm{ps}$ at the temperature studied here $(T=570 \mathrm{~K})$ [35]. Note that the typical time-step in a CG dynamic simulation is $0.01 \tau$, thus roughly 0.3 ps. For $N=20$ the atomistic simulations only covered a bead motion up to about the monomer size. This time mapping unit was used in figure $25.5 \mathrm{a}$, which shows $S(Q, t) / S(Q, 0)$ for a $N=5$ and $N=20$ BPA-PC melt [12]. For each chain length two independent sets of data are shown; the first has been obtained after reinsertion of chemical details in long-time CG trajectories (symbols); the second has been obtained from separate detailed, all-atom simulations (lines). Data
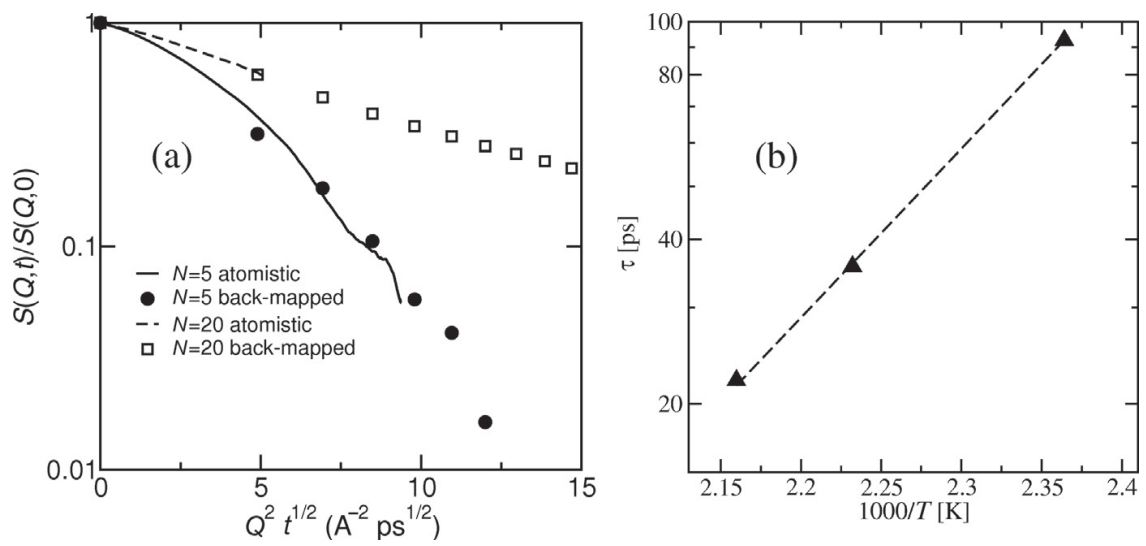

FIGURE 25.5 (a) Dynamic scattering function $S(Q, t) / S(Q, 0)$ of BPA-PC chains in the melt $(570 \mathrm{~K})$ as measured by n-spin echo experiments versus the scaled time $Q^{2} t^{1 / 2}$ for $Q=0.2 \AA^{-1}$ [12]. Data obtained by original atomistic simulations are shown by the solid and dashed line; data obtained from inverse mapped conformations are shown by the symbols. (b) Arrhenius representation of the time mapping constant for the ethylbenzene motions in PS melt [36]. 
are presented for $Q=0.2 \AA^{-1}$, which covers the typical chain extension. A remarkable agreement is observed by the data obtained based on the CG trajectory and the all-atom simulations. This perfect agreement of trajectories illustrates that the $\mathrm{CG}$ dynamic trajectories are physically meaningful down to very small length and time scales. It also shows that with such a time mapping of CG and atomistic simulations absolute data for long time and large scale dynamic quantities can be obtained without calibrating simulation timescales using experimental data. Based on the above time-mapping and inverse-mapping methods, the largest all-atom system simulated consisted of 200 BPA-PC chains of $N=120$ (corresponding to roughly 800,000 atoms in a box with a linear dimension of $100 \mathrm{~nm}$ ) up to $4 \times 10^{-5} \mathrm{sec}$.

\subsubsection{Dynamic Speedup: Additive Molecules in a Long-Chain Polystyrene Melt}

The above route to determining the physical time scale in a CG simulation has been applied to several systems. To better understand the physical origin of the dynamic speedup in comparison with all-atom models and real-life experimental systems, we discuss in this section an example of a simulation study of the dynamics of CG ethylbenzene (EB) molecules dissolved in a CG PS microstructure. A physical time scale was obtained by mapping the simulated EB diffusion coefficients onto the corresponding experimental data obtained by pulse field gradient NMR [36]. The time

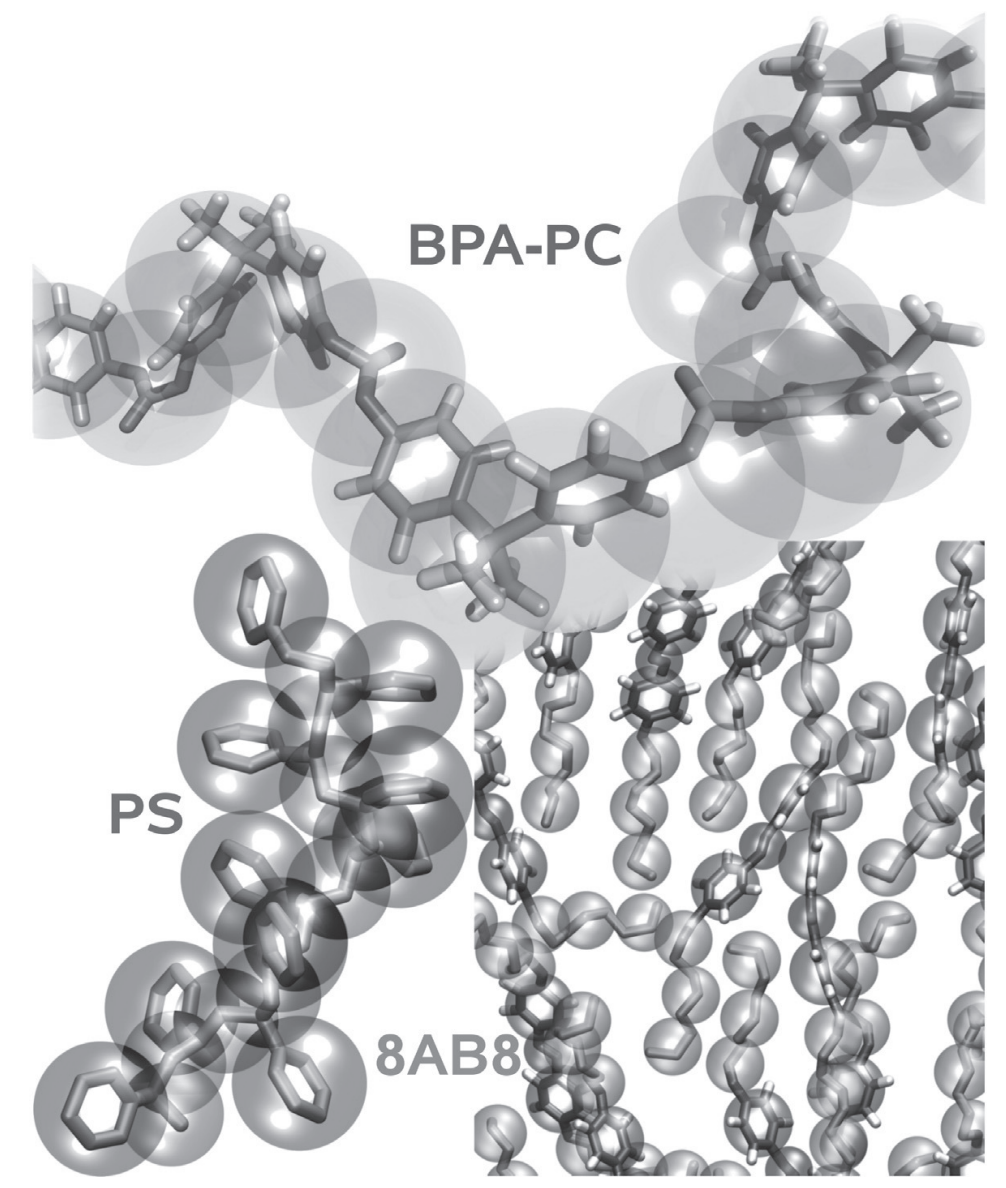

FIGURE 25.6 (See color insert following page XXX.) Snapshots of selected molecules from CG simulations of BPA-PC, PS, and 8AB8 indicating both CG centers and atomistic coordinates obtained through inverse mapping. 
conversion unit $\tau=D^{C G} / D^{\text {exp }}$ (expressed in picoseconds) is presented in figure 25.5b on a logarithmic scale versus the inverse temperature. The key observation is that $\tau$ depends exponentially on the temperature; that is, $\tau=\tau_{0} \exp (-A / T)$, where the constant $A$ is positive. This observation originates from the fact that energy barriers for EB diffusional motions are lower in the CG system where interparticle potentials are softer and more smoothly varying with distance. The time mapping $\tau(T)$ between the real and the CG system therefore follows an Arrhenius dependency with an "activation energy" $k_{\mathrm{B}} A$ describing an average reduction of energy barriers in the CG system. It should be noted that $D^{C G}$ and $D^{\exp }$ do not follow an Arrhenius dependency. Because the time scale for migration of the relatively large EB molecules is coupled to chain rearrangements of the PS matrix, it is important that the CG model is capable of reproducing the non-Arrhenius (VogelFulcher) type temperature dependence of structural relaxation of the melt.

\subsection{SOME RECENT DEVELOPMENTS AND FUTURE PERSPECTIVES}

\subsubsection{Adaptive Resolution MD}

In many systems formation (e.g., self-assembly) and dynamics of large-scale structures and conformations cannot be decoupled from local, chemical processes and specific intermolecular interactions. To perform computer simulations for those cases, dual-scale resolution schemes can be used [37-42]. One can however even go beyond using molecular models with fixed (single or dual) resolution and allow for a dynamic change of molecular resolution by changing the number of molecular DOF on-the-fly during the course of an MD simulation. Recently, such an adaptive resolution scheme (AdResS) has been introduced in which molecules can freely exchange between a high-resolution and low-resolution region [43-45]. A key ingredient in this new method is a transition region in which a weighting function is applied that mixes the high-resolution and low-resolution pair forces thereby slowly modifying the resolution of the molecules that move through [46]. The 'latent heat' associated with increasing or decreasing the number of molecular DOF is supplied or removed by a properly chosen thermostat. By these means thermodynamic equilibrium is maintained throughout the system. This method, which so far has been used for liquid water [44] and a polymer-solvent system [45], is of great interest in a much wider variety of systems. An example could be an active site on a protein where the biological function requires an explicit description of solvent molecules. It would clearly be beneficial if far away from the active site the system could be described at lower resolution to avoid spending $99 \%$ of computer time on moving water molecules around in regions not of primary interest.

\subsubsection{Surface Interactions of BIOMOLECULES}

Interactions of biomolecules with metal and inorganic surfaces are becoming increasingly important in nanobiotechnology. Typical questions involve how the functionality of a bio/inorganic hybrid device depends on the conformation of adsorbed biomolecules and how conformations are affected by the nature of the surface interactions involved. Multiscale modeling techniques that bridge between quantum, classical atomistic, and CG model descriptions are needed to approach such issues. Recently, initial steps have been made to bridge between the quantum and classical atomistic levels by performing a quantum-classical modeling of statistical conformations and interactions of amino acids and water molecules with metal surfaces [47]. This work has provided a recipe for treating surface interactions of amino acid residues in a classical-level description through an interactive quantum-classical modeling approach that can in principle be applied to larger organic molecules. Further progress will rely on the development of dual-resolution or adaptive resolution models that can be used to describe the system (solute and solvent) at high resolution close to the surface, combined with a description at lower resolution far away from the surface. 


\subsubsection{NONBONDED INTERACTIONS}

Although the iterative Boltzmann inversion method (equation (25.7)) provides nonbonded interaction potentials for CG models, it is based on radial distributions functions, which usually do not precisely define the system. In addition this can lead to very complicated and long-range potentials, which reduce the efficiency of the CG simulation significantly. Ideally one should aim to put as little as possible prior information into the model because that unavoidably leads to CG potentials that lack transferability and thus predictive potential. With respect to CG models for biopolymers in solution (e.g., oligopeptides) one ideally develops the CG force field in a way that distinguishes the bonded and nonbonded parts of the interaction potentials (in analogy to the method described above). Whereas for the bonded part, lessons learned from polymer coarse graining could be applied, for the nonbonded part important challenges remain. Current developments include empirical parameterization against thermodynamic data [48] and force-matching approaches [49,50]. Alternative to these approaches, intermolecular pair potentials of mean force obtained from atomistic MD simulations can be used. Based on this approach, CG potentials for aqueous electrolytes were recently reported [51,52]. This method has been extended to a wide range of electrolytes including, for example, alkylammonium salts for which a realistic description of the ion pairing and dissociation equilibrium requires accounting for aspects of hydrophobicity that-in addition to standard electrostatics—gives rise to an additional attraction between the ions [53].

\subsubsection{Perspectives}

Questions related to the specific systems discussed in this chapter lead automatically to another, almost philosophical aspect, namely-how specific is specific? In polymer physics one knows which properties are universal and which are chemistry specific. The systems considered there, however, are, in the end, very simple systems, where the above question is rather simple to answer. In problems related to structure formation, self-assembly, and surface interactions in synthetic and biological systems, specific interactions are operating. In these cases, it is far less understood which (chemistry) specific details should be kept in CG models (and which can safely be ignored). Moreover, it is not clear at what length scales the various CG modeling approaches described throughout this book merge and equally well describe these types of systems. Especially for biological molecules or complex structures employed in organic electronics, we, however, are still far away from such an understanding.

\section{ACKNOWLEDGMENTS}

We wish to acknowledge Berk Hess and Vagelis Harmandaris for providing data and figures. We wish to thank Berk Hess, Vagelis Harmandaris, Pim Schravendijk, Matej Praprotnik, and Luigi Delle Site for many stimulating discussions and fruitful collaborations. CP acknowledges financial support from the Volkswagen Foundation. Most atomistic simulations were carried out using the Gromacs simulation package [54]; CG simulations were mainly performed with the ESPResSo suit of programs [55].

\section{REFERENCES}

1. Tschöp, W., Kremer, K., Batoulis, J., Bürger, T., and Hahn, O. 1998. Simulation of polymer melts. I. Coarse-graining procedure for polycarbonates. Acta Polym. 49:61-74.

2. Tschöp, W., Kremer, K., Hahn, O., Batoulis, J., and Bürger, T. 1998. Simulation of polymer melts. II. From coarse-grained models back to atomistic description. Acta Polym. 49:75-79.

3. Müller-Plathe, F. 2002. Coarse-graining in polymer simulation: From the atomistic to the mesoscopic scale and back. ChemPhysChem 3:754-69.

4. Müller, M., Katsov, K., and Schick, M. 2006. Biological and synthetic membranes: What can be learned from a coarse-grained description? Phys. Rep. 434:113-76. 
5. Ayton, G. S., Noid, W. G., and Voth, G. A. 2007. Multiscale modeling of biomolecular systems: In serial and in parallel. Curr. Opin. Struct. Biol. 17:192-98.

6. Kremer, K. 2000. Computer simulations in soft matter science. In Soft and Fragile Matter, Nonequilibrium Dynamics, Metastability and Flow, ed. M. E. Cates and M. R. Evans, 145-84. Bristol: Institute of Physics.

7. Baschnagel, J., Binder, K., Doruker, P., Gusev, A. A., Hahn, O., Kremer, K., Mattice, W. L., Müller-Plathe, F., Murat, M., Paul, W., Santos, S., Suter, U. W., and Tries, V. 2000. Bridging the gap between atomistic and coarse-grained models of polymers: Status and perspectives. Adv. Polym. Sci. 152:41-156.

8. Müller-Plathe, F. 2003. Scale-hopping in computer simulations of polymers. Soft Mater. 1:1-31.

9. Murat, M., and Kremer, K. 1998. From many monomers to many polymers: Soft ellipsoid model for polymer melts and mixtures. J. Chem. Phys. 108:4340-48.

10. Bolhuis, P. G., Louis, A. A., Hansen, J. P., and Meijer, E. J. 2001. Accurate effective pair potentials for polymer solutions. J. Chem. Phys. 114:4296-311.

11. Abrams, K., and Kremer, K. 2003. Combined coarse-grained and atomistic simulation of liquid bisphenol A-polycarbonate: Liquid packing and intramolecular structure. Macromolecules 36:260-67.

12. Hess, B., León, S., Van der Vegt, N., and Kremer, K. 2006. Long time atomistic polymer trajectories from coarse grained simulations: Bisphenol-A polycarbonate. Soft Mater. 2:409-14.

13. Harmandaris, V. A., Adhikari, N. P., Van der Vegt, N. F. A., and Kremer, K. 2006. Hierarchical modeling of polystyrene: From atomistic to coarse-grained simulations. Macromolecules 39:6708-19.

14. Harmandaris, V. A., Reith, D., Van der Vegt, N. F. A., and Kremer, K. 2007. Comparison between coarse-graining models for polymer systems: Two mapping schemes for polystyrene. Macromol. Chem. Phys. 208:2109-20.

15. Peter, C., Delle Site, L., and Kremer, K. 2008. Classical simulations from the atomistic to the mesoscale and back: Coarse graining an azobenzene liquid crystal. Soft Matter 4:859-69.

16. Abrams, C. F., and Kremer, K. 2002. Effects of excluded volume and bond length on the dynamics of dense bead-spring polymer melts. J. Chem. Phys. 116:3162-65.

17. Weeks, J. D., Chandler, D., and Andersen, H. C. 1971. Role of repulsive forces in determining equilibrium structure of simple liquids. J. Chem. Phys. 54:5237-47.

18. Meyer, H., Biermann, O., Faller, R., Reith, D., and Müller-Plathe, F. 2000. Coarse graining of nonbonded inter-particle potentials using automatic simplex optimization to fit structural properties. J. Chem. Phys. 113:6264-75.

19. Press, W. H., Teukolsky, S. A., Vetterling, W. T., and Flannery, B. P. 1992. Numerical Recipes in C. The Art of Scientific Computing. Cambridge: Cambridge University Press.

20. Lyubartsev, A. P., and Laaksonen, A. 1995. Calculation of effective interaction potentials from radialdistribution functions: A reverse Monte-Carlo approach. Phys. Rev. E 52:3730-37.

21. Reith, D., Pütz, M., and Müller-Plathe, F. 2003. Deriving effective mesoscale potentials from atomistic simulations. J. Comp. Chem. 24:1624-36.

22. McCoy, J. D. and Curro, J. G. 1998. Mapping of explicit atom onto united atom potentials. Macromolecules 31:9362-68.

23. Auhl, R., Everaers, R., Grest, G. S., Kremer, K., and Plimpton, S. J. 2003. Equilibration of long chain polymer melts in computer simulations. J. Chem. Phys. 119:12718-28.

24. Santangelo, G., Di Matteo, A., Müller-Plathe F., and Milano, G. 2007. From mesoscale back to atomistic models: A fast reverse-mapping procedure for vinyl polymer chains. J. Phys. Chem. B 111:2765-73.

25. Hess, B., Peter, C., Özal, T. A., Van der Vegt, N. F. A. 2008. Fast-growth thermodynamic integration: Calculating excess chemical potentials of additive molecules in polymer microstructures. Macromolecules 41:2283-89.

26. Eilhard, J., Zirkel, A., Tschop, W., Hahn, O., Kremer, K., Scharpf, O., Richter, D., and Buchenau, U. 1999. Spatial correlations in polycarbonates: Neutron scattering and simulation. J. Chem. Phys. 110:1819-30.

27. Londono, J. D., Habenschuss, A., Curro, J. G., and Rajasekaran, J. J. 1996. Short-range order in some polymer melts from X-ray diffraction. J. Polym. Sci. B 34:3055-61.

28. Hahn, O., Mooney, D. A., Müller-Plathe, F., and Kremer, K. 1999. A new mechanism for penetrant diffusion in amorphous polymers: Molecular dynamics simulations of phenol diffusion in bisphenolA-polycarbonate. J. Chem. Phys. 111:6061-68.

29. Schmitz, H. 1999. Computersimulation von positronium-annihilation in polymeren. $\mathrm{PhD}$ thesis, University of Mainz, Germany.

30. Tozzini, V., Rocchia, W., and McCammon, J. A. 2006. Mapping all-atom models onto one-bead coarsegrained models: General properties and applications to a minimal polypeptide model. J. Chem. Theory Comput. 2:667-73. 
31. Kremer, K., and Grest, G. S. 1990. Dynamics of entangled linear polymer melts: A molecular-dynamics simulation. J. Chem. Phys. 92:5057-86.

32. Kremer, K. 2006. Polymer dynamics: Long time simulations and topological constraints. In Computer Simulations in Condensed Matter: From Materials to Chemical Biology, vol. 2. ed. M. Ferrario, G. Cicotti, and K. Binder, 341-78. Lect. Notes. Phys., vol. 704. Berlin, Heidelberg: Springer.

33. Padding, J. T., and Briels, W. J. 2002. Time and length scales of polymer melts studied by coarsegrained molecular dynamics simulations. J. Chem. Phys. 117:925-43.

34. Izvekov, S., and Voth, G. A. 2006. Modeling real dynamics in the coarse-grained representation of condensed phase systems. J. Chem. Phys. 125:151101.

35. León, S., Van der Vegt, N., Delle Site, L., and Kremer, K. 2005. Bisphenol A polycarbonate: Entanglement analysis from coarse-grained MD simulations. Macromolecules 38:8078-92.

36. Harmandaris, V. A., Adhikari, N. P., Van der Vegt, N. F. A., Kremer, K., Mann, B. A., Voelkel, R., Weiss, H., and Liew, C. 2007. Ethylbenzene diffusion in polystyrene: United atom atomistic/coarse grained simulations and experiments. Macromolecules 40:7026-35.

37. Chun, H. M., Padilla, C. E., Chin, D. N., Watanabe, M., Karlov, V. I., Alper, H. E., Soosaar, K., Blair, K. B., Becker, O. M., Caves, L. S. D., Nagle, R., Haney, D. N., and Farmer, B. 2000. MBO(N)D: A multibody method for long-time molecular dynamics simulations. J. Comput. Chem. 21:159-84.

38. Malevanets, A., and Kapral, R. 2000. Solute molecular dynamics in a mesoscale solvent. J. Chem. Phys. 112:7260-69.

39. Abrams, C. F., Delle Site, L., and Kremer, K. 2003. Dual-resolution coarse-grained simulation of the bisphenol-A-polycarbonate/nickel interface. Phys. Rev. E 67:021807.

40. Villa, E., Balaeff, A., Mahadevan, L., and Schulten, K. 2004. Multiscale method for simulating proteinDNA complexes. Multiscale Model. Simul. 2:527-53.

41. Delle Site, L., Leon, S., and Kremer, K. 2004. BPA-PC on a Ni(111) surface: The interplay between adsorption energy and conformational entropy for different chain-end modifications. J. Am. Chem. Soc. 126:2944-55.

42. Schravendijk, P., Van der Vegt, N., Delle Site, L., and Kremer, K. 2005. Dual-scale modeling of benzene adsorption onto $\mathrm{Ni}(111)$ and $\mathrm{Au}(111)$ surfaces in explicit water. Chemphyschem 6:1866-71.

43. Praprotnik, M., Delle Site, L., and Kremer, K. 2005. Adaptive resolution molecular-dynamics simulation: Changing the degrees of freedom on the fly. J. Chem. Phys. 123:224106.

44. Praprotnik, M., Matysiak, S., Delle Site, L., Kremer, K, and Clementi, C. 2007. Adaptive resolution simulation of liquid water. J. Phys. Condens. Mater 19:292201.

45. Praprotnik, M., Delle Site, L., and Kremer, K. 2007. A macromolecule in a solvent: Adaptive resolution molecular dynamics simulation. J. Chem. Phys. 126:134902.

46. Praprotnik, M., Kremer, K., and Delle Site, L. 2007. Fractional dimensions of phase space variables: A tool for varying the degrees of freedom of a system in a multiscale treatment. J. Phys. A: Math. Theor. 40:F281-88.

47. Schravendijk, P., Ghiringhelli, L., Delle Site, L., and Van der Vegt, N. F. A. 2007. Interaction of hydrated amino acids with metal surfaces: A multiscale modeling description. J. Phys. Chem. C 111:2631-42.

48. Marrink, S.-J., de Vries, A. H., and Mark, A. E. 2004. Coarse grained model for semiquantitative lipid simulations. J. Phys. Chem. B 108:750-60.

49. Izvekov, S., Parrinello, M., Burnham, C. J., and Voth, G. A. 2004. Effective force fields for condensed phase systems from ab initio molecular dynamics simulation: A new method for force-matching. J. Chem. Phys. 120:10896-913.

50. Izvekov, S., and Voth, G. A. 2005. A multiscale coarse-graining method for biomolecular systems. J. Phys. Chem. B 109:2469-73.

51. Hess, B., Holm, C., and Van der Vegt, N. F. A. 2006. Modeling multibody effects in ionic solutions with a concentration dependent dielectric permittivity. Phys. Rev. Lett. 96:147801.

52. Hess, B., Holm, C., and Van der Vegt, N. F. A. 2006. Osmotic coefficients of atomistic $\mathrm{NaCl}$ (aq) force fields. J. Chem. Phys. 124:164509.

53. Hess, B., and Van der Vegt, N. F. A. 2007. Solvent-averaged potentials for alkali-, earth alkali- and alkylammonium halide aqueous solutions. J. Chem. Phys. 127:234508.

54. Van der Spoel, D., Lindahl, E., Hess, B., Groenhof, G., Mark, A. E., and Berendsen, H. J. C. 2005. GROMACS: Fast, flexible, and free. J. Comput. Chem. 26:1701-18.

55. Limbach, H.-J., Arnold, A., Mann, B. A., and Holm, C. 2006. ESPResSo: An extensible simulation package for research on soft matter systems. Comput. Phys. Commun. 174:704-27. 